\title{
ON THE INVARIANT CHARACTER OF A SYSTEM OF PARTIAL DIFFERENTIAL EQUATIONS*
}

\author{
BY GORDON FULLER
}

1. Equations Set Up. We shall consider a differential equation

$$
\operatorname{div} F=|F|,
$$

where $F$ is a vector and $|F|$ denotes its length. Its real character is seen better if we write $u, v, w$ for the components of $F$ and $\rho$ for $|F|$; we have then the differential equation

$$
u_{x}+v_{y}+w_{z}=\rho,
$$

(subscripts throughout this discussion mean differentiation) with the condition

$$
u^{2}+v^{2}+w^{2}=\rho^{2},
$$

which shows that the relation imposed on $F$ is quadratic.

2. Auxiliary Quantities. In an attempt to reduce the system to linear equations we introduce auxiliary quantities $\alpha, \beta, \gamma, \delta$ (also functions of $x, y, z$ ), in terms of which we have

$$
\begin{aligned}
u & =2(\alpha \delta+\beta \gamma), & w & =\alpha^{2}+\beta^{2}-\gamma^{2}-\delta^{2}, \\
v & =2(\alpha \gamma-\beta \delta), & \rho & =\alpha^{2}+\beta^{2}+\gamma^{2}+\delta^{2} .
\end{aligned}
$$

Condition (3) is satisfied identically by a substitution of these expressions into it, and it remains to determine the conditions imposed upon $\alpha, \beta, \gamma, \delta$ by (2). Substitution gives

$$
\begin{aligned}
& \alpha\left(\delta_{x}+\gamma_{y}+\alpha_{z}-\frac{\alpha}{2}\right)+\beta\left(\gamma_{x}-\delta_{y}+\beta_{z}-\frac{\beta}{2}\right) \\
& +\gamma\left(\beta_{x}+\alpha_{y}-\gamma_{z}-\frac{\gamma}{2}\right)+\delta\left(\alpha_{x}-\beta_{y}-\delta_{z}-\frac{\delta}{2}\right)=0,
\end{aligned}
$$

* This paper was presented to the Society, April 8, 1932. It gives in an abbreviated form the material covered in the first part of a University of Michigan dissertation to be available in lithoprinted copies. 
which is a non-linear equation. However, if we equate to zero the coefficients of $\alpha, \beta, \gamma, \delta$, we obtain

$$
\begin{array}{ll}
\delta_{x}+\gamma_{y}+\alpha_{z}-\alpha / 2=0, & \beta_{x}+\alpha_{y}-\gamma_{z}-\gamma / 2=0, \\
\gamma_{x}-\delta_{y}+\beta_{z}-\beta / 2=0, & \alpha_{x}-\beta_{y}-\delta_{z}-\delta / 2=0,
\end{array}
$$

a set of linear equations, of which (5) and therefore (2) are consequences. It follows then, that if we know how to solve the linear system (6), we will have some solutions of the original system. However, we shall not be interested here in the solutions of equations but rather in interrelations of equations of different types. So far we have only found a linear system of which the given quadratic system is a consequence.

3. Generalization of Equations. We shall now investigate some peculiarities of this linear system. We observe in the first place that auxiliary quantities $\alpha, \beta, \gamma, \delta$ are used to represent the quantities $u, v, w, \rho$, of which only three are independent; we may expect then that there is some freedom in choosing these auxiliary quantities, and in fact we find without difficulty that we can replace $\alpha, \beta, \gamma, \delta$ by $\alpha^{\prime}, \beta^{\prime}, \gamma^{\prime}, \delta^{\prime}$, where

$$
\begin{array}{ll}
\alpha^{\prime}=\alpha \cos \Omega+\beta \sin \Omega, & \gamma^{\prime}=\gamma \cos \Omega-\delta \sin \Omega, \\
\beta^{\prime}=\alpha \sin \Omega+\beta \cos \Omega, & \delta^{\prime}=\gamma \sin \Omega+\delta \cos \Omega,
\end{array}
$$

without affecting the values obtained for $u, v, w, \rho$ by (4). The substitution of (7) changes the form of (6), however, unless $\Omega$ is constant. We prefer to consider the more general system

$$
\begin{aligned}
\gamma l-\delta m+\beta n+\delta_{x}+\gamma_{y}+\alpha_{z}-\alpha / 2 & =0, \\
-\delta l-\gamma m-\alpha n+\gamma_{x}-\delta_{y}+\beta_{z}-\beta / 2 & =0, \\
-\alpha l+\beta m+\delta n+\beta_{x}+\alpha_{y}-\gamma_{z}-\gamma / 2 & =0, \\
\beta l+\alpha m-\gamma n+\alpha_{x}-\beta_{y}-\delta_{z}-\delta / 2 & =0,
\end{aligned}
$$

in which $l, m, n$ are to be considered as given functions. The form of these equations is not changed under the transformations ( 7$)$; but the values of $l, m, n$ are affected and become respectively, ${ }^{*} l+\Omega_{x}, m+\Omega_{y}, n+\Omega_{z}$.

* This situation is parallel to that in the Dirac equations; $l, m, n$ correspond to the components of the four-potential. 
4. Transformations on the Functions. The original system of equations, (2) and (3), is invariant under coordinate transformations. That means that if we change the coordinates and at the same time subject the components of the vector $F$ to corresponding transformations, and leave $\rho$ as it was, the equations will have the same form. Is the system (8) invariant in the same sense? We will say in general that a system of equations is invariant under a transformation of coordinates, if for every transformation of coordinates $\Sigma$ we may introduce a transformation $T$ on the functions appearing in the system so that a simultaneous application of $\Sigma$ and $T$ gives a new system equivalent to the old one. In the case of the system (2), (3) these transformations $T$ are obtained easily; $u, v, w$, being the components of a vector, change in the same way as do the coordinates. The functions $l, m, n$ were introduced into (8) with the idea of absorbing $\Omega_{x}, \Omega_{y}, \Omega_{z}$; since $\Omega$ is an angle, a scalar function, $\Omega_{x}$, $\Omega_{y}, \Omega_{z}$ are components of its gradient, and we are led to assign transformations to $l, m, n$, where we regard them as components of a vector. But the quantities $\alpha, \beta, \gamma, \delta$ are neither scalars nor components of a vector-they are quantities of a special kind. However, transformations $T$ have been found for them.* They may be described in the following way. A general rotation of coordinate axes may be presented as the result of a succession of rotations each of which affects only two coordinates; corresponding to each of these we indicate below a transformation of the $\alpha, \beta, \gamma, \delta$. Namely,

$$
\begin{aligned}
& x y \text { plane rotation } \begin{cases}\alpha^{\prime}=\alpha c+\beta s, & \gamma^{\prime}=\gamma c+\delta s, \\
\beta^{\prime}=-\alpha s+\beta c, & \delta^{\prime}=-\gamma s+\delta c,\end{cases} \\
& x z \text { plane rotation } \begin{cases}\alpha^{\prime}=\alpha c+\delta s, & \gamma^{\prime}=-\beta s+\gamma c, \\
\beta^{\prime}=\beta c+\gamma s, & \delta^{\prime}=-\alpha s+\delta c,\end{cases} \\
& y z \text { plane rotation } \begin{cases}\alpha^{\prime}=\alpha c-\gamma s, & \gamma^{\prime}=\alpha s+\gamma c, \\
\beta^{\prime}=\beta c+\delta s, & \delta^{\prime}=-\beta s+\delta c,\end{cases}
\end{aligned}
$$

where $c$ and $s$, in each case, are the cosine and sine of one-half the angle through which the coordinate plane is rotated. Of

* Analogous formulas for the four-dimensional case were indicated by Darwin, Wave equation of electron, Proceedings of the Royal Society of London, (A), vol. 118, p. 656 . 
course these transformations on $\alpha, \beta, \gamma, \delta$ are not the only ones which satisfy our requirements, for they could be combined with (7), since (7) does not change the form of (8).

5. Elimination of $\alpha, \beta, \gamma, \delta$. The quantities $\alpha, \beta, \gamma, \delta$ and the expressions (4) were introduced to aid in the study of $u, v, w, \rho$, and consequently we attempt to find now the equations corresponding to (8) in terms of $u, v, w, \rho$. The divergence of a vector and the components of the curl are two standard types of expressions which appear in invariant systems. The divergence already appears in (2) and now we try to obtain the desired equations by aid of the components of the curl of $F$. These components may be had directly from (4) in terms of $\alpha, \beta, \gamma, \delta$ and their derivatives; then by use of (8) they may be easily expressed as follows:

$$
\begin{aligned}
& w_{y}-v_{z}=2\left(-\alpha \beta_{x}+\beta \alpha_{x}+\gamma \delta_{x}-\delta \gamma_{x}+\rho l\right), \\
& u_{z}-w_{x}=2\left(-\alpha \beta_{y}+\beta \alpha_{y}+\gamma \delta_{y}-\delta \gamma_{y}+\rho m\right), \\
& v_{x}-u_{y}=2\left(-\alpha \beta_{z}+\beta \alpha_{z}+\gamma \delta_{z}-\delta \gamma_{z}+\rho n\right) .
\end{aligned}
$$

We see that the left sides contain only derivatives of $u, v, w$, while $\rho, l, m, n$, and $\alpha, \beta, \gamma, \delta$ and their derivatives appear in the right members. Now we shall try to get rid of $\alpha, \beta, \gamma, \delta$ and their derivatives. To accomplish this we return to (4) and solve for $\alpha, \beta, \gamma, \delta$. We cannot, however, solve for $\alpha, \beta, \gamma, \delta$ in terms of $u, v$, $w$, and $\rho$ alone, for among $u, v$, $w$, and $\rho$ there are only three independent quantities. Hence a parameter must enter. From the last two expressions of (4) we obtain

$$
\alpha^{2}+\beta^{2}=\frac{\rho+w}{2}, \quad \gamma^{2}+\delta^{2}=\frac{\rho-w}{2},
$$

and therefore

$$
\begin{array}{lll}
\alpha=\left(\frac{\rho+w}{2}\right)^{1 / 2} \cos \phi, & \gamma=\left(\frac{\rho-w}{2}\right)^{1 / 2} \sin \psi, \\
\beta=\left(\frac{\rho+w}{2}\right)^{1 / 2} \sin \phi, & \delta=\left(\frac{\rho-w}{2}\right)^{1 / 2} \cos \psi,
\end{array}
$$

where $\phi$ and $\psi$ are not yet determined. Substituting these expressions into the first two of (4), we obtain 


$$
u=\left(\rho^{2}-w^{2}\right)^{1 / 2} \cos (\psi-\phi), \quad v=\left(\rho^{2}-w^{2}\right)^{1 / 2} \sin (\psi-\phi) .
$$

Therefore

$$
\tan (\psi-\phi)=\frac{v}{u}, \quad \text { or } \quad \psi-\phi=\arctan \frac{v}{u} .
$$

Let us select for our parameter the quantity $\psi+\phi$ and denote it by

$$
\theta=\psi+\phi
$$

We may express $\psi-\phi$ in terms of $u, v, w, \rho$, as shown by (11), but $\psi+\phi$ or $\theta$ cannot be so expressed. To see that this is true we have but to substitute in (4) the expressions as given by (10) for $\alpha, \beta, \gamma, \delta$, and taking into account (11), notice that (4) is satisfied for any value of $\theta$. Now if we substitute in the right members of equations (9), remembering (11) and (12), we obtain

$$
\begin{aligned}
& w_{y}-v_{z}=-\rho \theta_{x}+w\left(\arctan \frac{v}{u}\right)_{x}+2 \rho l, \\
& u_{z}-w_{x}=-\rho \theta_{y}+w\left(\arctan \frac{v}{u}\right)_{y}+2 \rho m, \\
& v_{x}-u_{y}=-\rho \theta_{z}+w\left(\arctan \frac{v}{u}\right)_{z}+2 \rho n .
\end{aligned}
$$

6. Invariance of the New System. We now have the set (13) which does not involve $\alpha, \beta, \gamma, \delta$. To show that it is equivalent to (8) we have but to replace $u, v, w, \rho$, and $\theta$ by their values in terms of $\alpha, \beta, \gamma, \delta$ by aid of (4) and (10), and notice that the set (8) is thus obtained. Since (8) is invariant we suspect that (13) is also invariant, even though $\theta$, which is entirely arbitrary, is present. Suppose then that we test it for invariance under our definition of an invariant system of equations. That is, we ask, whether or not it is possible, given (13) and a new system obtained from (13) by a transformation of coordinates, to arrive at (13) from the new set by taking combinations of this new set. Corresponding to the new set of equations is the set obtained from (8) by the same transformation. In the same way that we obtain (8) from (13) we may get the new set correspond- 
ing to (8) from the new set corresponding to (13). But we have seen above that (8) and its transformed set is the same, and further that (13) may be had from (8). Therefore (13) transforms in to the same set and is invariant in spite of the quantity $\theta$.

7. Elimination of $\theta$. The system (13) is not quite satisfactory. In the first place the components $u, v, w$ do not appear in a symmetric way; and in the second place it involves the quantity $\theta$, which, although it does not affect the invariant character of the system, is an unorthodox quantity. We shall see that by eliminating $\theta$ we obtain a system which can easily be made symmetric in $u, v, w$. Solving (13) for $\theta_{x}, \theta_{y}$, and $\theta_{z}$ we have, after rearranging the terms, from $\theta_{x y}=\theta_{y x}, \theta_{y z}=\theta_{z y}$, and $\theta_{x z}=\theta_{z x}$ :

$$
\begin{aligned}
& \rho\left(p_{y}-q_{x}\right)-\left(\rho_{y} p-\rho_{x} q\right)+2 \rho^{2}\left(m_{x}-l_{y}\right) \\
& \quad=\left(\rho w_{y}-\rho_{y} w\right)\left(\arctan \frac{v}{u}\right)_{x}+\left(\rho_{x} w-\rho w_{x}\right)\left(\arctan \frac{v}{u}\right)_{y}, \\
& \rho\left(q_{z}-r_{y}\right)-\left(\rho_{z} q-\rho_{y} r\right)+2 \rho^{2}\left(n_{y}-m_{z}\right) \\
& \quad=\left(\rho w_{z}-\rho_{z} w\right)\left(\arctan \frac{v}{u}\right)_{y}+\left(\rho_{y} w-\rho w_{y}\right)\left(\arctan \frac{v}{u}\right)_{z}, \\
& \rho\left(r_{x}-p_{z}\right)-\left(\rho_{x} r-\rho_{z} p\right)+2 \rho^{2}\left(l_{z}-n_{x}\right) \\
& \quad=\left(\rho w_{x}-\rho_{x} w\right)\left(\arctan \frac{v}{u}\right)_{z}+\left(\rho_{z} w-\rho w_{z}\right)\left(\arctan \frac{v}{u}\right)_{x},
\end{aligned}
$$

where $p, q, r$ are the components of the curl of $F, w_{y}-v_{z}, u_{z}-w_{x}$, $v_{x}-u_{y}$. Hence we have a set of equations, known to be invariant, where $\theta$ does not appear.

8. Invariance of Forms. We shall now try to express (14) in a more satisfactory form. The left member of the first equation may be written in the form

Now

$$
\rho^{2}\left[\left(\frac{p}{\rho}-2 l\right)_{y}-\left(\frac{q}{\rho}-2 m\right)_{x}\right] .
$$

$$
\left(\frac{p}{\rho}-2 l\right)_{y}-\left(\frac{q}{\rho}-2 m\right)_{x}
$$

is the $z$ component of the curl of a certain vector, namely, the vector $(1 / \rho)$ curl $F-2 L$, where $L=(l, m, n)$. Since curl is an 
invariant operation, this member is now in a satisfactory form. The right side must be the $z$ component of a vector, but its form does not show this fact. We may, however, change the form by using the relations

$$
\rho^{2}=u^{2}+v^{2}+w^{2}, \quad u_{x}+v_{y}+w_{z}=\rho,
$$

and relations obtained from these by differentiation. Multiplying the right side by $\rho / \rho$, we have

$$
\begin{aligned}
& \frac{1}{\rho}\left[\left(\rho \rho w_{y}-\rho \rho_{y} w\right)\left(\arctan \frac{v}{u}\right)_{x}\right. \\
& \left.\quad+\left(\rho \rho_{x} w-\rho^{2} w_{x}\right)\left(\arctan \frac{v}{u}\right)_{y}\right],
\end{aligned}
$$

and from

$$
\begin{gathered}
\rho^{2}=u^{2}+v^{2}+w^{2}, \quad \rho \rho_{y}=u u_{y}+v v_{y}+w w_{y}, \\
\rho \rho_{x}=u u_{x}+v v_{x}+w w_{x},
\end{gathered}
$$

this becomes

$$
\begin{aligned}
& \frac{1}{\rho}\left[\left(u^{2}+v^{2}+w^{2}\right) w_{y}-\left(u u_{y}+v v_{y}+w w_{y}\right) w\right]\left(\arctan \frac{v}{u}\right)_{x} \\
& \quad+\frac{1}{\rho}\left[\left(u u_{x}+v v_{x}+w w_{x}\right) w-\left(u^{2}+v^{2}+w^{2}\right) w_{x}\right]\left(\arctan \frac{v}{u}\right)_{y} \\
& =\frac{1}{\rho}\left[\left(u^{2}+v^{2}\right) w_{y}-\left(u u_{y}+v v_{y}\right) w\right]\left(\arctan \frac{v}{u}\right)_{x} \\
& \quad+\frac{1}{\rho}\left[\left(u u_{x}+v_{x}\right) w-\left(u^{2}+v^{2}\right) w_{x}\right]\left(\arctan \frac{v}{u}\right)_{y} \\
& =\frac{1}{\rho}\left[\begin{array}{ll}
\left.w_{y}\left(u v_{x}-v_{x}\right)-w_{x}\left(u v_{y}-v_{y}\right)-w\left(u_{y} v_{x}-u_{x} v_{y}\right)\right] \\
=
\end{array}\right. \\
& \frac{1}{\rho}\left|\begin{array}{lll}
u & v & w_{x} \\
u_{x} & v_{x} & w_{x} \\
u_{y} & v_{y} & w_{y}
\end{array}\right| .
\end{aligned}
$$

The right side appears here in a symmetric form. In a similar fashion we change the forms of the other two equations and we have then the following equations: 


$$
\begin{aligned}
& \rho^{3}\left[\left(\frac{q}{\rho}-2 m\right)_{z}-\left(\frac{r}{\rho}-2 n\right)_{y}\right]=\left|\begin{array}{lll}
u & v & w \\
u_{y} & v_{y} & w_{y} \\
u_{z} & v_{z} & w_{z}
\end{array}\right|, \\
& \rho^{3}\left[\left(\frac{r}{\rho}-2 n\right)_{x}-\left(\frac{p}{\rho}-2 l\right)_{z}\right]=\left|\begin{array}{lll}
u & v & w \\
u_{z} & v_{z} & w_{z} \\
u_{x} & v_{x} & w_{x}
\end{array}\right|, \\
& \rho^{3}\left[\left(\frac{p}{\rho}-2 l\right)_{y}-\left(\frac{q}{\rho}-2 m\right)_{x}\right]=\left|\begin{array}{lll}
u & v & w_{x} \\
u_{x} & v_{x} & w_{x} \\
u_{y} & v_{y} & w_{y}
\end{array}\right| .
\end{aligned}
$$

The left members of our equations are the $x, y, z$ components of a vector, and consequently in the right members we have a new kind of expression which like divergence and curl is obtained in invariant fashion. In fact, it can be verified by rotation of axes that the determinant expressions change in exactly the same way as do the components of a vector. We may inquire how our new equations change by the transformations (7). Since $u, v, w$, $\rho$, as we have observed, are unchanged it is obvious that the right members are unaffected. On the left side we have but to look for the effect on the terms involving the derivatives of $l, m, n$, all other terms being expressible in terms of $\rho$ and derivatives of $u, v, w, \rho$. But (7), as we have seen, adds $\Omega_{x}, \Omega_{y}$, $\Omega_{z}$, respectively, to $l, m, n$. We have then, noticing the left member of the last equation above, transformed $m_{x}-l_{y}$ into $\left(m+\Omega_{y}\right)_{x}$ $-\left(l+\Omega_{x}\right)_{y} ;$ and since $\Omega_{x y}=\Omega_{y x}$, we see that no change results. The other quantities are likewise invariant by (7). We now have our equations in forms which indicate their invariant character, and thus have accomplished the purpose of this discussion.

It might be added that the left members of the equations may be combined into one expression by using vector notation, and if we denote by $V$ the vector whose components are the determinants, the three equations may be written

$$
\rho^{3} \operatorname{curl}\left(\frac{1}{\rho} \operatorname{curl} F-2 L\right)=V .
$$

Sioux Falls College 